\title{
GEOMETRIA E ARITMÉTICA COMBINAM COM EQUAÇÕES DO 2 GRAU?
}

\author{
F. Quaranta, E. G. Lourenço, A. Alves e I. S. Medeiros \\ Laboratório de Matemática de João Câmara - Instituto Federal do Rio Grande do Norte \\ francisco.quaranta@ifrn.edu.br
}

Artigo submetido em outubro/2011 e aceito em dezembro/2013

\section{RESUMO}

Quando falamos em equação do segundo grau, é imediata a associação com a fórmula geral atribuída erroneamente à Bháskara no Brasil. Isto se deve ao uso exagerado e, muitas vezes, exclusivo dessa fórmula. $\mathrm{O}$ objetivo desse trabalho é incentivar e discutir a relevância e a abrangência de outros métodos de resolução de equações do segundo grau que atualmente tem sido deixado de lado pela maioria dos professores brasileiros, tais como: um método geométrico presente em "Os Elementos" de Euclides, o completamento de quadrados (onde demonstraremos a fórmula geral através do raciocínio geométrico - adição de áreas) e o método da soma e produto (no qual apresentaremos um artifício que amplia o seu uso para raízes fracionárias).

PALAVRAS-CHAVE: Equação de Segundo Grau, Completamento de Quadrados, Euclides, Soma e Produto.

\section{DO ARITHMETIC AND GEOMETRY COMBINE WITH QUADRATIC EQUATIONS?}

\begin{abstract}
When we talk about quadratic equation, it is immediate to associate with the general formula erroneously attributed to Bhaskara in Brazil. This is due to overuse and often unique to this formula. The objective of this paper is to encourage and discuss the relevance and scope of other methods for solving quadratic equations that currently has been left aside by most Brazilian
\end{abstract}

teachers, such as a geometric method present in "The Elements" of Euclid, the completion of squares (which demonstrate the general formula by geometric reasoning - adding areas) and the method of sum and product (which we will present in a device that extends its use for fractional roots).

KEYWORDS: quadratic equation, completing the square, Euclid, sum and product. 


\section{GEOMETRIA E ARITMÉTICA COMBINAM COM EQUAÇÕES DO 2 GRAU?}

\section{INTRODUÇÃO}

Muitas vezes, erramos ao achar que à medida que os avanços na ciência são obtidos, eles chegam automaticamente ao ensino. E pior que isso: essa substituição do novo conhecimento pelo antigo é sempre bem feita! Em outras palavras, temos uma falsa certeza que o ensino dos dias atuais é necessariamente melhor que o ensino de anos anteriores. No ensino de equação de 2 o grau, temos um bom exemplo desse questionável progresso.

Hoje, quando pensamos em resolver uma equação do tipo $x^{2}-5 x+6=0$, associamos automaticamente aquela fórmula geral $\mathrm{x}=\frac{-b \pm \sqrt{b^{2}-4 a c}}{2 a}$ (indevidamente chamada no Brasil por "Fórmula de Bhaskara). Infelizmente, sofremos um processo de redução progressiva das diferentes abordagens que podem ser escolhidas pelo professor no ensino das equações do segundo grau. Uma vez que essa solução tem caráter totalmente abrangente, achou-se adequado abandonar as outras formas de se apresentar e de se resolver equações do segundo grau. $O$ ensino atual resume-se ao estudo com enfoque exclusivamente algébrico.

Este artigo intenciona oferecer um material didático com abordagens e soluções de equações de segundo grau que não se limitem a uma formula e ao enfoque algébrico. Ele faz parte de um futuro livro que propõe soluções de caráter aritmético e geométrico das equações de segundo grau. Queremos oferecer ao professor e ao aluno mais curioso um texto que permita o aprendizado de outros métodos de resolução. Alguns deles, inclusive, em determinados casos, podem oferecer um ganho expressivo de tempo na obtenção das raízes da equação, uma vez que apresentam maior adequação e praticidade.

Três métodos de resolução de equações de segundo grau serão desenvolvidos neste artigo. O primeiro é um método geométrico desenvolvido por Euclides por volta do século III a.C. e presente na sua famosa obra "Os Elementos". Ele se destina a determinado grupo de equações e usa exclusivamente as ferramentas dos gregos, sem qualquer uso de álgebra. O segundo será o método do completamento de quadrados que combina argumentos algébricos e geométricos. É uma opção abrangente, permitindo inclusive a dedução da conhecida fórmula geral. $O$ terceiro método é o da soma e do produto das raízes. Ele possui uma essência aritmética, mas tem uma abrangência limitada (apenas raízes inteiras). Será apresentado um artifício que permite ampliálo para raízes racionais.

\section{MÉTODO GEOMÉTRICO (EUCLIDES)}

As equações de segundo grau possuem uma longa História. Os primeiros registros vêm dos Mesopotâmicos e dos Egípcios que resolviam alguns casos, sob a forma de receitas. Resolviam problemas que podem ser transformados em equações com formatos bem específicos.

Os gregos desenvolveram soluções geométricas dando um caráter mais estruturado às soluções, através da discussão da sua validade. Discutiram os vários subgrupos que podiam organizar os problemas que geravam as equações. Os árabes e hindus estruturaram ainda mais as soluções, ainda recorrendo à geometria, mas introduziram alguns símbolos, apesar da linguagem ainda retórica. 
Apenas com o francês Viéte, no início da idade moderna, que é introduzido o simbolismo algébrico, forma pela qual reconhecemos atualmente as equações do segundo grau. Somente a partir daí, é que estavam criadas todas as condições para o surgimento de uma fórmula geral para todas as equações de 20 grau. Mas apenas no século XIX, quando os números negativos e complexos foram aceitos e elevados à categoria de números por Argand e Gauss, é que todas as soluções das equações passaram a ser definitivamente reconhecidas. Por ter prevalecido durante a maior parte da História da Matemática, a resolução das equações de 20 grau através de métodos geométricos não pode ser esquecida! Optamos pela construção proposta por um matemático de notória relevância: o grego Euclides.

\section{1 - Resolução geométrica proposta por Euclides}

Vamos apresentar o método de Euclides através de um problema que usa uma linguagem que se aproxima da forma como as equações de segundo grau eram apresentadas na sua época. A geometria era a ferramenta de formulação dos problemas e consequentemente a incógnita, ou seja, o número que se desejava descobrir era sempre um segmento que seria obtido através da régua e do compasso.

\subsubsection{Problema Motivador}

Deseja-se achar dois lados de um retângulo cujo perímetro mede $80 \mathrm{~cm}$ e cuja área vale $256 \mathrm{~cm}^{2}$.

\subsubsection{Solução}

Primeiramente, vale destacar que esse problema pode ser convertido para outro formato. Em linguagem atual, os lados desse retângulo que queremos achar são chamados de $x$ e $y$. Logo, podemos escrever duas equações com duas variáveis: $\left\{\begin{array}{l}x+y=40 \\ x \cdot y=256\end{array}\right.$

Neste instante, podemos fazer outra formulação para o mesmo problema: achar dois números cuja soma $(b)$ e produto $\left(c^{2}\right)$ são conhecidos. Usando o enfoque atual, tentamos resolver esse sistema. Pelo método da substituição, obtemos uma equação do segundo grau.

$$
y=40-x \quad(40-x) \cdot x=256 \quad x^{2}-40 x+256=0
$$

Assim, necessitamos resolver uma equação do segundo grau. Transformamos assim a forma de apresentação de um problema da linguagem tipicamente grega para a linguagem dita moderna.

\subsection{Solução de Euclides}

A solução que propomos a seguir está presente na sua obra-prima "Os Elementos".

Ela é adequada para o que hoje chamamos de equações do segundo grau que possuam o seguinte formato: $\boldsymbol{x}^{2}+\boldsymbol{c}^{2}=\boldsymbol{b} \boldsymbol{x}$, onde $\boldsymbol{b}$ e $\boldsymbol{c}^{2}$ representam números comensuráveis (inteiros e fracionários positivos). Vale ressaltar que na sua época, a Geometria era a única forma de se fazer matemática. A Álgebra nem sonhava em aparecer. Assim, $\boldsymbol{b}$ significava um comprimento de um segmento e $\boldsymbol{c}^{2}$ a área de um quadrado de lado $\boldsymbol{c}$. Assim, apenas os números positivos eram admitidos. 


\subsubsection{Passo a passo da solução}

1 - Constrói-se o segmento $A B$ de comprimento $\boldsymbol{b}$ e o ponto médio $C$.

2 - Faz-se a mediatriz de $A B$.

3 - Nela, marca-se o ponto $O$ de forma que $C O$ seja igual ao lado $c$ do quadrado de área conhecida.

4 - Com centro em $O$, traça-se o arco do círculo de raio $\frac{b}{2}$. Marca-se D na interseção do arco com $A B$.

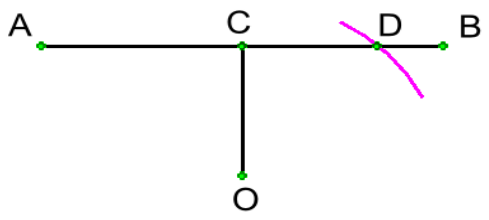

Figura 1 - Figura final do passo a passo da solução de Euclides

As soluções do problema (e consequentemente da equação) são os segmentos $A D$ e $D B$.

\subsection{Justificativa para a solução}

Euclides justifica a validade da construção por uma proposição (II.5) do seu livro. Nela, utiliza equivalência de áreas. Faremos outra justificativa, mais curta e adaptada à linguagem atual. Aplicando Pitágoras no triângulo DCO, o cateto CD será:

$$
\begin{aligned}
& \boldsymbol{C D}=\sqrt{b^{2} / 4-c^{2}}=\sqrt{40^{2} / 4-256}=\sqrt{400-256}=\sqrt{144}=12 \\
& \sqrt[c]{\int_{0}^{b / 2}}
\end{aligned}
$$

Figura 2 - Triângulo DCO formado a partir da figura 1

Assim,

$$
\begin{aligned}
& \boldsymbol{A D}=\boldsymbol{A C}+\boldsymbol{C D}=\frac{\boldsymbol{b}}{\mathbf{2}}+\sqrt{b^{2} / 4-c^{2}}=20+\mathbf{1 2}=\mathbf{3 2} \\
& \boldsymbol{D} \boldsymbol{B}=C \boldsymbol{B}-C D=\frac{\boldsymbol{b}}{\mathbf{2}}-\sqrt{b^{2} / 4-c^{2}}=\mathbf{2 0 - 1 2}=\mathbf{8}
\end{aligned}
$$

Fazendo a verificação da soma e do produto das raízes obtidas, podemos confirmar a veracidade dos resultados. Assim, retornando ao sistema do problema motivador $\boldsymbol{A D}$ $=\boldsymbol{x}=\mathbf{3 2}$ e $\boldsymbol{D} \boldsymbol{B}=\boldsymbol{y}=\mathbf{8}$. Se substituirmos esses valores na equação: $\boldsymbol{x}^{2}+\boldsymbol{c}^{2}=\boldsymbol{b} \boldsymbol{x}$, teremos a equação satisfeita em cada caso. 


\subsection{Existência das Raízes}

Vale ressaltar que nesse tipo de equação do segundo grau, as raízes só existirão se $\boldsymbol{b}>2 \boldsymbol{c}$. Isso é fácil de ver tanto na solução geométrica quanto na algébrica. Na primeira, o arco de comprimento $\frac{b}{2}$ só cruzará $\boldsymbol{A} \boldsymbol{B}$ se ele pelo menos igualar $\boldsymbol{c}^{2}$. No segundo caso, o radicando $\frac{b}{2}-c^{2}$ será negativo, o que é impossível para uma raiz quadrada.

\subsection{Sinal das raízes}

Nesse caso de equação de segundo grau, as raízes, se existirem, sempre serão positivas, uma vez que sempre serão as partes do segmento $\boldsymbol{A} \boldsymbol{B}$.

\subsection{Abrangência do método}

Como foi dito desde o início, ele foi feito para a resolução de problemas que se transformam em equações do tipo $\boldsymbol{x}^{2}+\boldsymbol{c}^{2}=\boldsymbol{b} \boldsymbol{x}$.

Obs.: com algumas adaptações e colocação de um par de eixos cartesianos, podemos ampliá-lo de forma tal que resolve qualquer tipo de equação do segundo grau de forma $a x^{2}+b x+c=$ 0 .

\section{FORMULA GERAL ATRAVÉS DO COMPLETAMENTO DE QUADRADOS}

A seguir, apresentaremos um método, conhecido como completamento de quadrados, que contém tanto a abordagem geométrica quanto algébrica. Ele será usado para a demonstração da fórmula geral.

Para compreensão do método, serão utilizadas figuras, uma vez que ele usa idéias geométricas e as transforma em argumentos algébricos e vice-versa.

\subsection{Resolvendo equações do segundo grau por completamento de quadrados}

\subsubsection{Exemplo 1}

Vamos resolver a equação $2 x^{2}+8 x+2=0$. Para facilitar o processo de completamento de quadrados e transformar a expressão em um trinômio quadrado perfeito, iremos inicialmente multiplicar a equação pelo coeficiente a, no caso 2 . A nova equação será: $4 x^{2}+16 x+4=0$

Como vimos anteriormente, podemos representar cada termo desta equação por uma figura geométrica. Se extrairmos a raiz do elemento $4 x^{2}$ encontraremos $2 x$ que será transformado em um quadrado:

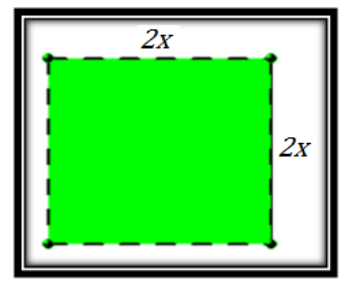

Figura 3 - Quadrado maior de lado $2 x$. 
O elemento $16 x$ é quebrado em dois retângulos congruentes de lados $2 x$ e 4 :

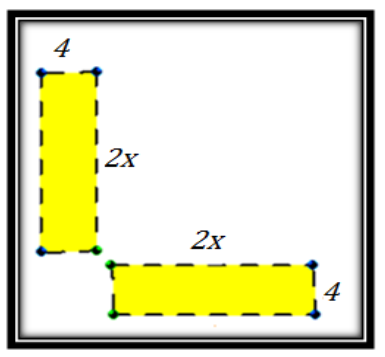

Figura 4 - Retângulos formados a partir do termo $16 x$.

Se unirmos o quadrado maior de lado $2 x$ com os dois retângulos perceberemos que, para formar um quadrado perfeito, precisamos de um quadrado menor de lado 4 e área 16 para completar o quadrado. Com isso, podemos concluir que o quadrado que faltará para completar o trinômio sempre terá lado igual a $\frac{b}{2 a}$. Neste caso, como $b=16$ e $a=2$, o lado vale 4 .

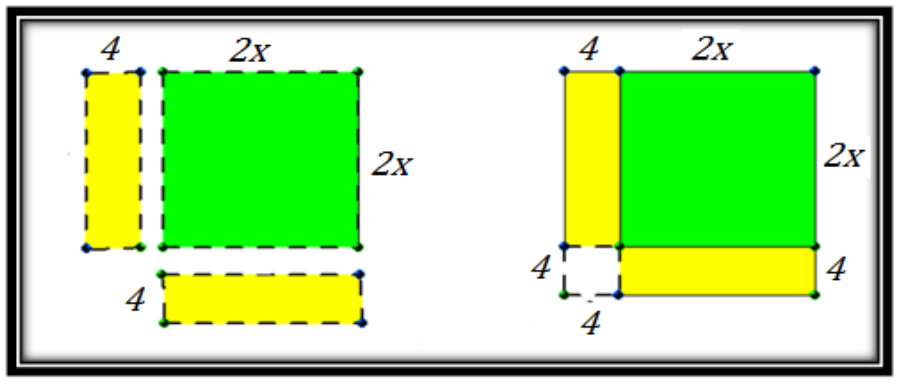

Figura 5 - Soma dos monômios

Para descobrir o número que somaremos aos dois membros da equação para gerar um trinômio quadrado perfeito, basta fazer uma subtração entre a área do quadrado menor e o termo independente. Assim, $16-4=12$.

Somando 12 aos membros da equação, encontramos um trinômio quadrado perfeito:

$$
4 x^{2}+16 x+16=12
$$

Fatoramos o trinômio:

$$
(2 x+4)^{2}=12
$$

Extraímos a raiz dos membros da equação:

$$
2 x+4= \pm \sqrt{12}
$$

Isolamos o elemento $x$ subtraindo 2 de seus membros:

As raízes da equação são:

$$
x=\frac{-4 \pm 2 \sqrt{3}}{2}
$$

$$
x=\left\{\begin{array}{c}
-\sqrt{3}-2 \\
\text { ou } \\
\sqrt{3}-2
\end{array}\right.
$$

\subsection{A Fórmula geral}

No $9^{\circ}$ ano do ensino fundamental, quando estudamos equações do segundo grau, o método de resolução mais usado pelos professores é a fórmula geral. Pelo fato de sua demonstração ser longa e complicada, ela é raramente apresentada. 
Com ajuda do método de completamento de quadrados, podemos justificar de maneira simples o procedimento algébrico adotado na sua demonstração.

Finalmente apresentaremos a fórmula geral que pode resolver qualquer tipo de equação do segundo grau!

\subsection{Demonstração}

Se tivermos uma equação do tipo $a x^{2}+b x+c=0$ podemos associá-la a um trinômio quadrado perfeito. Para tanto, devemos usar o artifício algébrico de multiplicar toda a equação por um termo que viabilize tal associação, por exemplo, $4 a$ (podem ser outros). A nova equação ficará:

$$
\begin{gathered}
4(a x)^{2}+4 a b x+4 a c=0 \\
(2 a x)^{2}+2(2 a x) b+4 a c=0
\end{gathered}
$$

Podemos representar o elemento $(2 a x)^{2}$ como um quadrado de lado $2 a x$ :

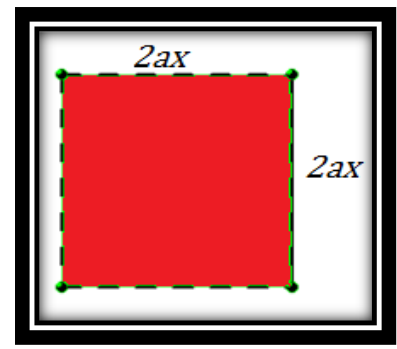

Figura 6 - Quadrado formado a partir da multiplicação da equação por $4 a$

Já o elemento $4 a b x$ pode ser dividido em duas partes e representado por dois retângulos congruentes de lados $2 a x$ e $b$.

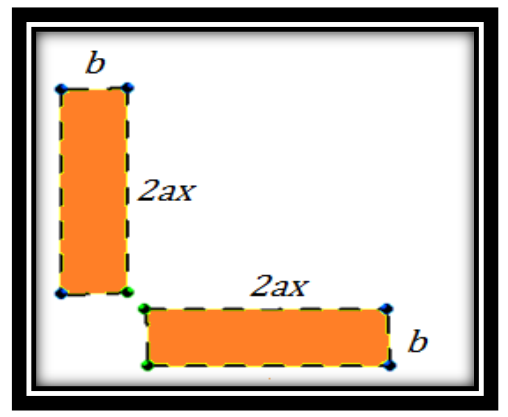

Figura 7 - Retângulos formados a partir da multiplicação da equação por $4 a$

Se unirmos o quadrado vermelho maior com os retângulos laranjas poderemos perceber que para completar um quadrado maior, será necessário somar um quadrado menor de lado $b$ e área $b^{2}$ igual à, a diferença entre $b^{2}-4 a c$ é o número que precisaremos somar aos membros da equação para poder transformá-la em um trinômio quadrado perfeito.

Então somaremos $b^{2}-4 a c$ aos membros da equação.

$$
\begin{gathered}
(2 a x)^{2}+2(2 a x) b+4 a c+b^{2}-4 a c=b^{2}-4 a c \\
(2 a x)^{2}+2(2 a x) b+b^{2}=b^{2}-4 a c
\end{gathered}
$$


Encontrado o trinômio quadrado perfeito faremos a seguir o mesmo procedimento feito anterior mente.

Fatoramos o trinômio quadrado perfeito:

$$
[2 a x+b]^{2}=b^{2}-4 a c
$$

Extraímos as raízes dos membros da equação:

$$
[2 a x+b]= \pm \sqrt{b^{2}-4 a c}
$$

Subtraímos $b$ dos seus membros:

$$
[2 a x]=-b \pm \sqrt{b^{2}-4 a c}
$$

Dividimos os dois membros por $2 a$ :

$$
x=\frac{-b \pm \sqrt{b^{2}-4 a c}}{2 a}
$$

O termo $b^{2}-4 a c$ é denominado discriminante. Recebe como símbolo a letra grega delta $\Delta$. Ele merece uma denominação especial uma vez que ajuda a analisar a natureza numérica das raízes.

Eis as soluções genéricas de uma equação de segundo grau:

$$
x=\frac{-b \pm \sqrt{\Delta}}{2 a}
$$

\subsection{Abrangência do método:}

O método do completamento de quadrados resolve, a princípio, qualquer equação de segundo grau. Ou seja, é o método mais abrangente apresentado neste artigo e pode resolver qualquer deste tipo de equação inclusive aquelas que possuem raízes complexas! Que no caso da fórmula geral acontecerá quando o discriminante for negativo. Não quer dizer, entretanto, que sua solução é sempre imediata. Discutiremos a seguir, grupos de equações que possuem uma resolução mais rápida pelo método do completamento.

O grupo de resolução mais imediata acontece quando já temos um trinômio quadrado perfeito. Veja o exemplo:

$$
x^{2}+10 x+25=0
$$

Veja que a equação acima já é um trinômio quadrado perfeito. Neste caso, nós fatoramos o trinômio e fazemos os mesmos procedimentos feitos anteriormente.

$$
\begin{gathered}
(x+5)^{2}=0 \\
x+5=\sqrt{0} \\
x=-5
\end{gathered}
$$

Outros casos não imediatos, mas ainda assim rápidos é quando temos uma equação onde o coeficiente $\boldsymbol{a}$ é um quadrado perfeito e $\boldsymbol{b}$ é múltiplo do dobro de $\sqrt{\boldsymbol{a}}$. Neste caso, temos as medidas dos lados dos retângulos números inteiros. Podemos afirmar que ao multiplicar toda a 
equação por $\mathbf{4 a}$ estaremos garantindo a inexistência de fração nos lados dos retângulos. Um caso onde podemos observa isso com clareza é na demonstração da fórmula geral.

\section{EQUAÇÃO DO SEGUNDO GRAU - SOMA E PRODUTO}

\subsection{Apresentação}

No segundo método apresentado neste artigo, desenvolvemos uma fórmula geral que pode resolver os problemas que envolvam equação do segundo grau com uma incógnita, conhecido indevidamente no Brasil como fórmula de Bháskara. No entanto, esta solução se apresenta na maioria das situações, lenta e ao mesmo tempo trabalhosa, evidenciando então, a necessidade de soluções alternativas para casos específicos.

No momento de escolher quais equações de segundo grau serão trabalhadas com seus alunos, percebemos que os professores possuem uma tendência por aquelas que contenham raízes inteiras. Isso é compreensível, já que existe uma barreira cognitiva no aprendizado dos números fracionários e irracionais. Porém, este uso se torna excessivo por parte do professor produzindo uma limitação na visão do aluno. Um exemplo claro dessa limitação ocorre quando observamos a reação frequente de um professor e consequentemente do aluno ao se deparar com um discriminante que não seja um quadrado perfeito. Em função desse vício de utilização de soluções inteiras das equações, o método a ser apresentado a seguir possui a característica de resolvê-las rapidamente, de maneira simples e prática.

O método da soma e produto é um caminho aritmético, mas estruturado com formato algébrico, onde sua solução é construída a partir da procura das possíveis combinações, que se encaixam nos valores da soma e produto das raízes.

A partir da fórmula geral, serão deduzidas as relações de soma e produto e em seguida serão apresentados exemplos.

\subsection{O Método}

Sendo $x=\frac{-b \pm \sqrt{b^{2}-4 a c}}{2 a}$ a fórmula geral, podemos determinar a soma e produto das raízes da seguinte maneira:

Se denotarmos a soma das raízes $x_{1}+x_{2}$ por $S$ e o produto das raízes $x_{1} \cdot x_{2}$ por $P$, então poderemos provar que $S=-\frac{b}{a}$ e $P=\frac{c}{a}$.

\subsection{Demonstração}

Como $x_{1}=\frac{-b+\sqrt{\Delta}}{2 a}$ e $x_{2}=\frac{-b-\sqrt{\Delta}}{2 a}$, podemos escrever a soma S será:

Logo:

$$
S=\frac{-b+\sqrt{\Delta}}{2 a}+\frac{-b-\sqrt{\Delta}}{2 a}
$$

$$
S=\frac{-b+\sqrt{\Delta}-b-\sqrt{\Delta}}{2 a}=-\frac{2 b}{2 a}=-\frac{b}{a}
$$

$\mathrm{O}$ mesmo procedimento para o produto $\mathrm{P}$ das raízes: 


$$
P=\frac{-b+\sqrt{\Delta}}{2 a} \cdot \frac{-b-\sqrt{\Delta}}{2 a}
$$

Desenvolvendo o produto da soma pela diferença de dois termos:

$$
P=\frac{b^{2}-\Delta}{2 a} \text {. }
$$

Mas $\Delta=b^{2}-4 a c$, logo:

$$
P=\frac{b^{2}-\left(b^{2}-4 a c\right)}{4 a^{2}}=\frac{4 a c}{4 a^{2}}=\frac{c}{a}
$$

\subsection{Abrangência do método}

Após concluir que a soma das raízes pode ser obtida por $-\frac{b}{a}$ e o produto por $\frac{c}{a}$, é perceptível que se as raízes da equação do $2^{\circ}$ grau forem inteiras, teremos obrigatoriamente os coeficientes b e c múltiplos do coeficiente a. A recíproca, porém, não é verdadeira, ou seja, se b e c são múltiplos de a, não podemos garantir que as raízes são inteiras.

Portanto, mesmo que tenhamos os coeficientes b e c múltiplos de $a$, teremos uma pequeníssima probabilidade de existirem raízes inteiras caso b e c sejam escolhidos aleatoriamente. Porém se quisermos estender esse método para raízes racionais, o artifício a seguir permitirá tal ampliação.

\subsection{Raízes fracionárias}

Já vimos anteriormente que resolver equações usando soma e produto tem se tornado muito útil hoje em dia, devido à constante utilização por parte dos professores de equações de segundo grau que possuem raízes inteiras nas atividades desenvolvidas em sala de aula.

No entanto, ao nos depararmos com equações que contém soluções fracionárias normalmente tendemos a não utilizar a soma e produto como alternativa de resolução. Veremos agora que mesmo para equações fracionárias, com um pouco de manipulação, esse método pode continuar a ter bastante utilidade.

Imagine que você se depare com a seguinte equação:

$$
2 x^{2}-7 x+6=0
$$

Afirmamos aqui que essa equação fica mais fácil de ser resolvida por soma e produto se multiplicarmos o coeficiente de $x^{2}(a=2)$ pelo termo independente $(c=6)$. Ao fazermos isso, criamos a equação:

$$
x^{2}-7 x+12=0
$$

Suas raízes tem como soma 7 e produto 12 , ou seja, elas valem 4 e 3 .

Podemos verificar que 4 e 3 não são as raízes da equação proposta inicialmente, mas elas são dadas por $\frac{4}{2=a}=2 \mathrm{e} \frac{3}{2=a}$.

Vamos justificar nossa afirmação.

A equação $a x^{2}+b x+c=0$ tem soluções determinadas por 


$$
x=\frac{-b \pm \sqrt{b^{2}-4 a c}}{2 a}
$$

Enquanto que a equação obtida quando tornamos o coeficiente a unitário e geramos um novo termo independente dado pelo produto de a por $c$. Assim a nova equação de variável independente $y$ será:

$$
y^{2}+b y+a c=0
$$

Suas soluções são determinadas por:

$$
y=\frac{-b \pm \sqrt{b^{2}-4 a c}}{2.1}
$$

Fica perceptível que as duas soluções diferem apenas no seu denominador (a primeira com fator $\boldsymbol{a}$ e a segunda sem o fator $\boldsymbol{a}$ ). Portanto, se acharmos as soluções da segunda e dividirmos por $\boldsymbol{a}$ achamos as soluções da primeira.

$$
\frac{y}{a}=x
$$

Concluindo, sempre que nos depararmos com equações quadráticas cujas soluções sejam fracionárias podemos tentar encontrá-las com mais facilidade se multiplicarmos o coeficiente de $x^{2}$ pelo termo independente gerando o novo coeficiente $c^{\prime}=a$. $c$. Acharmos as soluções desta nova equação e dividirmos essas soluções pelo coeficiente de $x^{2}$.

Quadro explicativo:

- A equação $a x^{2}+b x+c=0$ ( com soluções $x_{1}$ e $x_{2}$ )

- $y^{2}+b y+a c=0$ (equação obtida quando multiplicamos a por c, com soluções $y_{1} e$ $\left.\mathrm{y}_{2}\right)$

- $\mathrm{x}_{1}=\frac{y 1}{a}$ e $\mathrm{x}_{2}=\frac{y 2}{a}$

\section{CONCLUSÃO}

A diversidade de métodos para resolução das equações de segundo grau permite aos alunos uma visão geral e conectada da matemática. Eles serão capazes de relacionar e compreender melhor os conceitos apresentados em cada método. Com o andamento da pesquisa, foi possível notar o relacionamento harmonioso que há entre os métodos, onde alguns conceitos se complementam: como é o caso da fórmula geral que pode ser deduzida através do uso de completamento de quadrados. Neste trabalho, também foi abordada uma solução não convencional, proposta por Euclides em seu livro "Os Elementos", que permite uma noção mais concreta do conceito de raiz da equação (no caso, um segmento). A visão que se têm hoje de uma raiz é de um objeto algébrico, que não se pode ver, portanto o conhecimento deste método amplia o repertório conceitual de quem o conhece.

Notou-se também que os processos de resolução se tornaram mais imediatos, como por exemplo, o uso das relações de soma e produto que tornam a resolução relativamente simples, para alguns casos. Quando as raízes da equação são fracionárias, apresentamos um método pouco utilizado e extremamente rápido. Isto amplia de forma significativa a quantidade de equações de segundo grau que podem ser resolvidas de forma acelerada. 
Conforme dissemos anteriormente, este artigo é um recorte de um material mais amplo e que aprofunda no objetivo de aumentar o conhecimento dos professores e alunos sobre as equações de segundo grau. Essa omissão de outros métodos não compromete, entretanto o nosso maior objetivo, visto que o material presente já pode ser suficiente para estimular os alunos a investigar e pesquisar mais sobre a área.

\section{REFERÊNCIAS}

EUCLIDES. Os Elementos. Tradução de Irineu Bicudo. 1a edição. São Paulo: Ed. UNESP, 2009.

ROQUE, Tatiana, História da matemática: Uma Visão Crítica, Desfazendo Mitos e Lendas. Rio de Janeiro: Ed. Zahar, 2012.

PITOMBEIRA, João Bosco. A História da equação do 2 o grau. 1a edição. Rio de Janeiro: Ed. UFRJ, 2004.

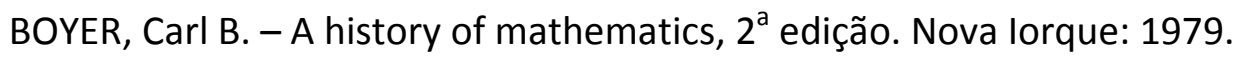

CHASLES, Michel - Aperçu historique sur l'origine et le développement des méthodes en géométrie. Bruxelas: 1837. 